\title{
Role of the solar wind magnetic field in the interaction of a non-magnetized body with the solar wind: An electromagnetic 2-D particle-in-cell simulation
}

\author{
Tomoko Nakagawa ${ }^{1}$ and Shinya Kimura ${ }^{2}$ \\ ${ }^{1}$ Tohoku Institute of Technology, 35-1 Yagiyama Kasumi-cho, Taihaku-ku, Sendai, Miyagi 982-8577, Japan \\ ${ }^{2}$ NTT Software Corporation, Teisan Kannai Bldg. 4F, 209, Yamasitatyo, Nakaku, Yokohama, Kanagawa 231-8551, Japan
}

(Received September 13, 2010; Revised January 31, 2011; Accepted February 9, 2011; Online published September 7, 2011)

\begin{abstract}
The solar wind interaction with a non-magnetized, electrically non-conducting body is studied using a twodimensional electromagnetic full particle simulation. The solar wind magnetic field is introduced into the simulation scheme as an initial condition together with the electric field generated by the motion of the solar wind. The solar wind magnetic field controls the direction of the thermal flow of the electrons and causes an asymmetry of the negative charging of the downstream-side surface. The negative charging and the potential drop are largest at the position where the solar wind magnetic field is perpendicular to the surface of the nonmagnetized body. In the absence of photoelectrons, the solar wind electrons begin to be expelled by the negative charging at the terminator and then flow away along the field line producing streaks of enhancements of the electron density.
\end{abstract}

Key words: Wake, surface charging, electric potential, solar wind magnetic field, non-magnetized, electrically non-conducting body, 2D electromagnetic PIC simulation.

\section{Introduction}

The solar wind interaction with a non-magnetized body is quite different from that which occurs in the case of the Earth. In the absence of a global magnetic field, the solar wind plasma can directly access a body or its atmosphere.

The interaction processes depend on the scale size of the body, the presence or absence of an ionosphere and the electric conductivity of the surface of the body. In this paper, the solar wind interaction with a non-magnetized, non-conducting body having no ionosphere is studied using a two-dimensional electromagnetic full particle simulation. This analysis might be applicable to the Moon or asteroids.

The solar wind interaction with a non-magnetized body such as the Moon is characterized by particle absorption and surface charging (Freeman and Ibrahim, 1975). The solar wind particles that hit the Moon are absorbed by the surface, creating a plasma cavity called the lunar wake behind the Moon (Schubert and Lichtenstein, 1974, and references therein). Because the electron thermal speed is higher than the solar wind speed, the nightside surface of the Moon is hit only by electrons and becomes negatively charged (Colwell et al., 2007, and references therein). The negative charging of the nightside surface of the Moon was observed by the Lunar Prospector (Halekas et al., 2002, 2003, 2005, 2008).

Recent observations by Kaguya and Chandrayaan have

Copyright (C) The Society of Geomagnetism and Earth, Planetary and Space Sciences (SGEPSS); The Seismological Society of Japan; The Volcanological Society of Japan; The Geodetic Society of Japan; The Japanese Society for Planetary Sciences; TERRAPUB.

doi:10.5047/eps.2011.02.006 provided new findings concerning the Moon (e.g., Saito et al., 2008; Tsunakawa et al., 2010; Futaana et al., 2010). Among these are several phenomena which indicate asymmetries associated with the direction of the interplanetary magnetic field (IMF). Nishino et al. (2009a, b) found two types of intrusion of solar wind protons into the central wake near the Moon due to the Larmour motion of the protons together with the inward electric field at the wake boundary (type I), and with the solar-wind pickup of the scattered protons at the dayside lunar surface (type II), both of which show strong asymmetry controlled by the direction of the IMF. Futaana et al. (2010) reported a Chandrayaan1 observation of another type of proton entry into the near wake along the magnetic field in the solar wind frame of reference.

It has also been found by Kaguya plasma wave observation (LRS/WFC-H) that there exists an asymmetric structure of electron density profile at the wake boundary depending on the direction of the IMF. The electron density is often enhanced at the wake boundary of the northern hemisphere when the $B_{z}$ component of the anti-sunward IMF is positive (that is, directed northward). The electron density enhancement occurs on the southern hemisphere when the $B_{z}$ component of the anti-sunward IMF is negative (directed southward). The north-south asymmetry reverses for the sunward IMF (Kasahara et al., personal communication, 2010).

To understand the phenomena that are considered to be controlled by the solar wind magnetic field, it would be helpful to examine the basic role of the interaction of the solar wind magnetic field with non-magnetized bodies us- 
Table 1. Choice of parameters.

\begin{tabular}{clccccccc}
\hline Run & $v_{\mathrm{e}} / c$ & $\lambda_{\mathrm{D}} / R_{\mathrm{O}}$ & $\Delta x / \lambda_{\mathrm{D}}$ & $\Omega_{\mathrm{e}} / \omega_{\mathrm{e}}$ & $\phi_{\mathrm{B}}$ & $r_{\mathrm{iL}} / R_{\mathrm{O}}$ & $r_{\mathrm{eL}} / R_{\mathrm{O}}$ & $\Delta t$ \\
\hline$\# 1$ & 0.1 & 0.25 & $1 / 3.2$ & 12 & $-45^{\circ}$ & 0.94 & 0.029 & 0.005 \\
$\# 2$ & 0.1 & 0.25 & $1 / 3.2$ & 12 & $-15^{\circ}$ & 0.94 & 0.029 & 0.005 \\
$\# 3$ & 0.05 & 0.125 & $1 / 1.6$ & 12 & $-45^{\circ}$ & 0.47 & 0.014 & 0.005 \\
$\# 4$ & 0.05 & 0.125 & $1 / 1.6$ & 0.75 & $-45^{\circ}$ & 7.54 & 0.23 & 0.016 \\
\hline
\end{tabular}

ing numerical simulations.

To deal with the solar wind interaction with a nonmagnetized, non-conducting body on which surface charging plays an important role, it is desirable to treat the electrons as particles. Particle-in-cell codes have been used to study solar wind interaction with non-magnetized obstacles by several authors (Farrell et al., 1998; Birch and Chapman, 2001, 2002; Guio and Pécseli, 2004, 2005), but they did not include surface charging in their simulation because their interests focused rather on the infilling of the wake, the ion acceleration, or the phase space structures in the wake.

Kimura and Nakagawa (2008) included surface charging in their 2-dimensional, full-particle electromagnetic code to calculate the electric field at the wake boundary. They succeeded in reproducing the ambipolar electric field at the wake boundary, the ion acceleration into the central void, the surface charging of the nightside surface, and the intense electric field at the terminator simultaneously in a selfconsistent manner, but they did not include photoemission or the solar wind magnetic field.

In this paper, the solar wind magnetic field is included in the electromagnetic full particle simulation. The effects of photoelectrons, although they are of crucial importance, are not included in order to concentrate on the role of the magnetic field. The crustal magnetic fields and the solar wind protons reflected by the lunar surface (Saito et al., 2008) give rise to a variety of interesting phenomena, but in this paper we limit ourselves to the basic cases of a non-magnetized body immersed in the solar wind having an intrinsic velocity distribution.

\section{Numerical Simulation}

\subsection{Two-dimensional electromagnetic PIC simulation}

A 2-D, full-particle electromagnetic code (Birdsall and Langdon, 1985) is used in this study. The simulator is the same as that used by Kimura and Nakagawa (2008), with the exception that it now allows for inclusion of the solar wind magnetic field.

We solve the equation of motion of $7.86 \times 10^{6}$ ions and as many electrons that are initially distributed over a $20 R_{\mathrm{O}} \times$ $20 R_{\mathrm{O}}$ simulation box $\left(-5 R_{\mathrm{O}}<x<15 R_{\mathrm{O}},-10 R_{\mathrm{O}}<y<\right.$ $10 R_{\mathrm{O}}$, where $R_{\mathrm{O}}$ is the radius of the obstacle ) except for the inside of the obstacle. The initial velocity distributions are shifted Maxwellian

$$
f_{\mathrm{i}}(\mathbf{v})=\frac{n_{\mathrm{i}}}{\pi^{\frac{3}{2}} v_{\mathrm{i}}^{3}} \exp \left(-\frac{\left(v_{x}-v_{\mathrm{sw}}\right)^{2}+v_{y}^{2}+v_{z}^{2}}{v_{\mathrm{i}}^{2}}\right)
$$

for ions and

$$
f_{\mathrm{e}}(\mathbf{v})=\frac{n_{\mathrm{e}}}{\pi^{\frac{3}{2}} v_{\mathrm{e}}^{3}} \exp \left(-\frac{\left(v_{x}-v_{\mathrm{sw}}\right)^{2}+v_{y}^{2}+v_{z}^{2}}{v_{\mathrm{e}}^{2}}\right)
$$

for electrons, where $v_{\mathrm{sw}}$ is the solar wind speed and $v_{\mathrm{i}}$ and $v_{\mathrm{e}}$ are the thermal speeds of ions and electrons, respectively. We set the ratio $v_{\mathrm{i}}: v_{\mathrm{sw}}: v_{\mathrm{e}}=1: 8: 32$. As the simulation starts, the plasmas flow down the simulation domain due to the anti-sunward bulk velocity $\left(v_{\mathrm{sw}}, 0,0\right)$, forming the plasma cavity behind the obstacle. The particles that leave the simulation box at the downstream end are removed from the simulation domain.

The particles that collide with the obstacle are also removed from the simulation box after giving their electric charge to the surface of the obstacle. The electric charges are fixed to each position of the collision, on the assumption that the obstacle is electrically insulating. No emission of secondary electrons is considered.

On removal of these particles, as many particles are injected from the upstream boundary. To reduce the time for the calculation, we use a high-speed solar wind, $v_{\mathrm{sw}}=$ $0.025 c$ (or $0.0125 c$ ), together with the electron thermal speed $v_{\mathrm{e}}=0.1 c$ (or $0.05 c$ ), which leads to a Debye length $\lambda_{\mathrm{D}} \equiv v_{\mathrm{e}} / \sqrt{2} \omega_{\mathrm{p}}$ as large as $0.25 R_{\mathrm{O}}$ (or $0.125 R_{\mathrm{O}}$ ), where $\omega_{\mathrm{p}}$ is the plasma frequency. The Debye length is typically of the order of 10-100 $\mathrm{m}$ in the average solar wind, so it should be noted that not all the results of the present simulation can be applied directly to a large obstacle such as the Moon whose radius is $10^{4}$ to $10^{5}$ times as large as the Debye length. The scaling of this simulation is rather more suitable for a smaller object such as an asteroid.

It should also be noted that the effects of photoelectrons are not included in this simulation. Thus, we cannot discuss the electric potential on the dayside surface of the obstacle. It might also change the electric field structure at the terminator.

Table 1 summarizes the parameters for 4 simulation runs. Details of the simulation scheme are fully described in Kimura and Nakagawa (2008).

\subsection{Inclusion of the solar wind magnetic field}

A uniform magnetic field $\mathbf{B}=\left(B \cos \theta_{\mathrm{B}}, B \sin \theta_{\mathrm{B}}, 0\right)$ is defined at $256 \times 256$ grids over the simulation domain as an initial condition, where $\theta_{\mathrm{B}}$ is the angle between the magnetic field and the solar wind flow along the $x$ axis. We start with $\theta_{\mathrm{B}}=-45^{\circ}$ to represent the direction of the average solar wind magnetic field.

The magnitude of the magnetic field is chosen so that the ion Larmour radius $r_{\mathrm{iL}}$ is smaller than the radius of the obstacle $R_{\mathrm{O}}$. As $r_{\mathrm{iL}}=v_{\mathrm{i}} / \Omega_{\mathrm{i}}=32 v_{\mathrm{e}} / \Omega_{\mathrm{e}}$ and $R_{\mathrm{O}}=$ $4 \lambda_{\mathrm{D}}\left(\right.$ or $8 \lambda_{\mathrm{D}}$ ) in our simulation, we require an electron cyclotron frequency $\Omega_{\mathrm{e}}$ larger than $8 \sqrt{2} \omega_{\mathrm{p}}$ (or $4 \sqrt{2} \omega_{\mathrm{p}}$ ). Consequently, the Alfvén speed becomes larger than the speed of light $c$ and any magnetic distortion propagates faster than the speed of light. So it should be noted that we cannot discuss the deformation of the magnetic field in 

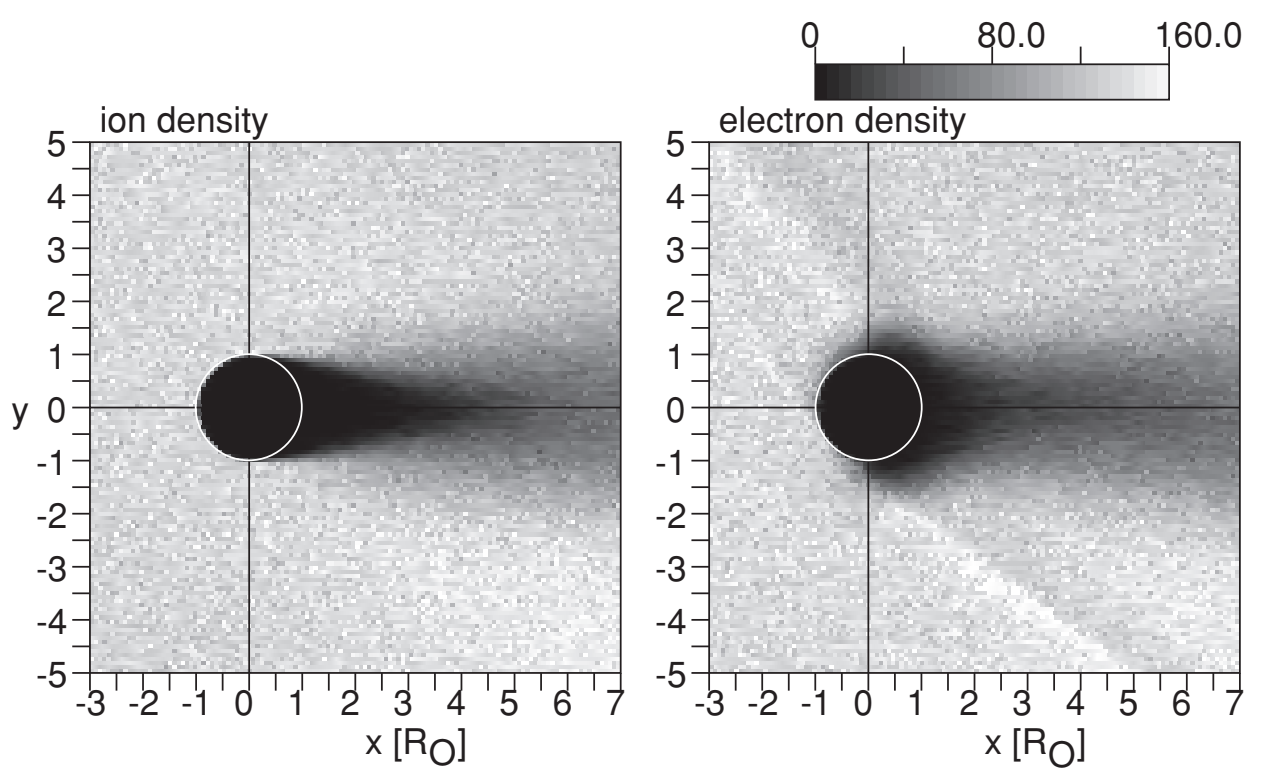

Fig. 1. Two-dimensional plots of the ion density $n_{\mathrm{i}}$ (left) and the electron density $n_{\mathrm{e}}$ (right) around the obstacle (outlined with a white circle) for the simulation run \#1. The solar wind flows from the left to the right. The solar wind magnetic field is $-45^{\circ}$ from the $x$-axis.

this simulation. We employ $\Omega_{\mathrm{e}} / \omega_{\mathrm{p}}=12$ in simulation runs $\# 1$ to $\# 3$, and then relaxed the condition to $\Omega_{\mathrm{e}} / \omega_{\mathrm{p}}=0.75$ in simulation run \#4.

The time step $\Delta t$ is set so that it satisfies the Courant condition and is smaller than $10^{-2}$ times the electron cyclotron period $2 \pi / \Omega_{\mathrm{e}}$.

In order to reproduce the frozen-in condition of the solar wind, the electric field $\mathbf{E}=-\mathbf{V}_{\mathrm{sw}} \times \mathbf{B}$ generated by the motion of the solar wind magnetic field past the obstacle is introduced as an initial condition. Otherwise, the plasmas cannot flow with the solar wind magnetic field but are guided by the external magnetic field. After that, the magnetic field and the electric field are calculated by solving Maxwell's equations with the FDTD method.

\section{Result}

\subsection{Plasma structure}

Figure 1 shows the ion and electron densities around the non-magnetized obstacle in the solar wind magnetic field with $\theta_{\mathrm{B}}=-45^{\circ}$, obtained from simulation run $\# 1$. The Debye length is $0.25 R_{\mathrm{O}}$ and the ion Larmour radius is $0.94 R_{\mathrm{O}}$ for this run. This is a snapshot at the time $t=$ $4.5 \times 10^{2} \omega_{\mathrm{p}}^{-1}$ (90000 steps), when the solar wind has swept the simulation domain twice.

An ion void is formed behind the obstacle. The ions cannot reach the downstream-side surface of the obstacle because the thermal speed is smaller than the bulk speed of the solar wind. The ion density structure is nearly symmetric with respect to the $x$-axis. Compared with the case with no magnetic field (figure 1 of Kimura and Nakagawa, 2008), no influence of the magnetic field is recognized in Fig. 1.

The electrons, whose thermal speed is much higher than the solar wind bulk speed, can reach the downstream-side surface of the obstacle. The negative charge accumulating on the surface expels the following electrons, creating an electron void. The mechanism is the same as in the non- magnetized solar wind, but the difference is that the electrons can go upstream only along the magnetic field lines, due to the small Larmour radius $r_{\mathrm{eL}}$. The number flux of the electrons per unit area of the surface of the obstacle is large at the position where the magnetic field is perpendicular to the surface. As a result, the charge density is thought to be larger in the negative- $y$ region, producing a larger area of depressed electron density than in the positive- $y$ region.

In addition to the asymmetry of the electron void, enhancements of electron density were found streaking from the vicinity of the terminator. In the right panel of Fig. 1, a ridge of electron density enhancement runs from $(x, y)=$ $(0.5,-2)$ to $(4.5,-5)$ in units of $R_{\mathrm{O}}$, accompanied by a slight depression next to the enhancement. On the positive$y$ side, a faint but broad enhancement extends from $(0,1.5)$ to $(-3,5) R_{\mathrm{O}}$ upstream. It should be noted that the streaks extend far beyond the Debye length. The streaks are not parallel to the magnetic field. The angle between the ridges of the electron density and the solar wind direction (along the $x$-axis) is $-37^{\circ}$ in the negative- $y$ region deviating by 8 degrees from the solar wind direction, and $131^{\circ}$ in the positive- $y$ region, with 4 degrees of deviation.

Figure 2 shows the bulk velocities of ions and electrons averaged over about 120 particles in each bin. The motion of ions, whose Larmour radius is $0.94 R_{\mathrm{O}}$, is not affected by the presence of the solar wind magnetic field. Because of their low thermal speed, it takes time for the ions to get to the center of the void, whilst they are convected away by the solar wind. Thus, they cannot reach the downstream-side surface of the obstacle and the central wake region near the obstacle is completely void of ions.

In contrast, electrons are found in the center of the wake near the obstacle surface, except for a part of the negative$y$ area. In the positive- $y$ side of the central void, the bulk speed is sometimes very high.

Upstream of the obstacle, the bulk velocity of the electrons is nearly the same as the convection velocity of the 

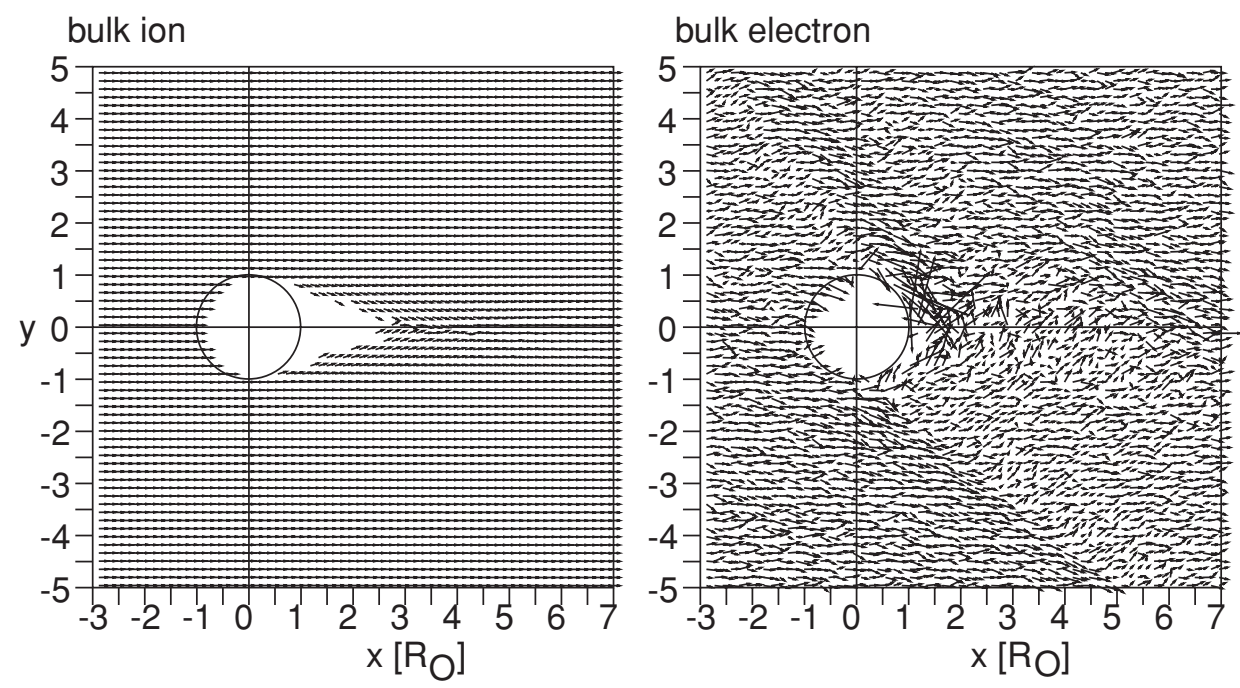

Fig. 2. Bulk velocities of ions and electrons averaged over each bin of $256 \times 256$ cells. The results from simulation run $\# 1$.

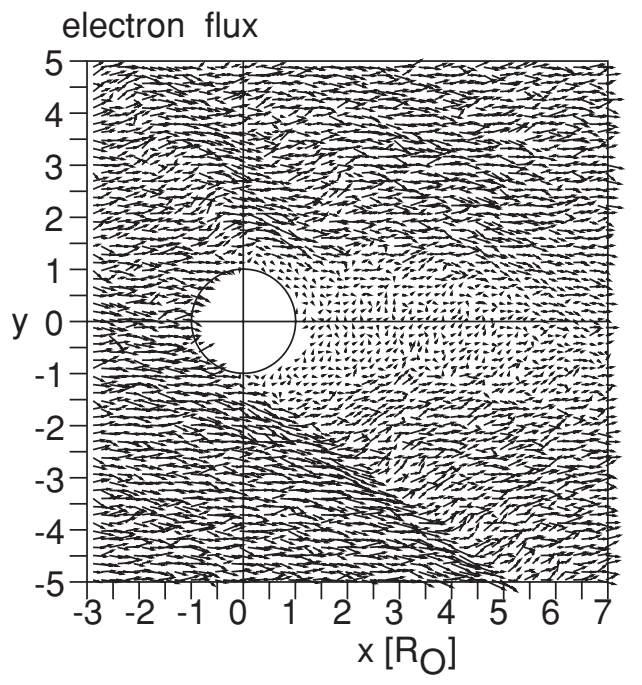

Fig. 3. Flux of the electrons. The result of the simulation run \#1.

solar wind. They begin to be skewed away at the terminator of the obstacle being expelled by the negative charges on the downstream-side surface of the obstacle. The expelled electrons flow away along the magnetic field being convected by the motion of the magnetic field, producing the streaks of enhancement of the electron density as seen in Fig. 1.

Figure 3 shows the flux of the electrons. The electrons expelled away from the terminator on the negative- $y$ side carry significant flux. On the positive- $y$ side, the effect of the expelled electrons is not very large. It is also found that the high speed electrons in the positive- $y$ region of the central void as seen in Fig. 2 do not contribute much electron flux into the void because of their low number density.

\subsection{Electric field structure}

Figure 4 shows the electric potential calculated with the relaxation method from the electric field and charge density obtained from the simulation run \#1.

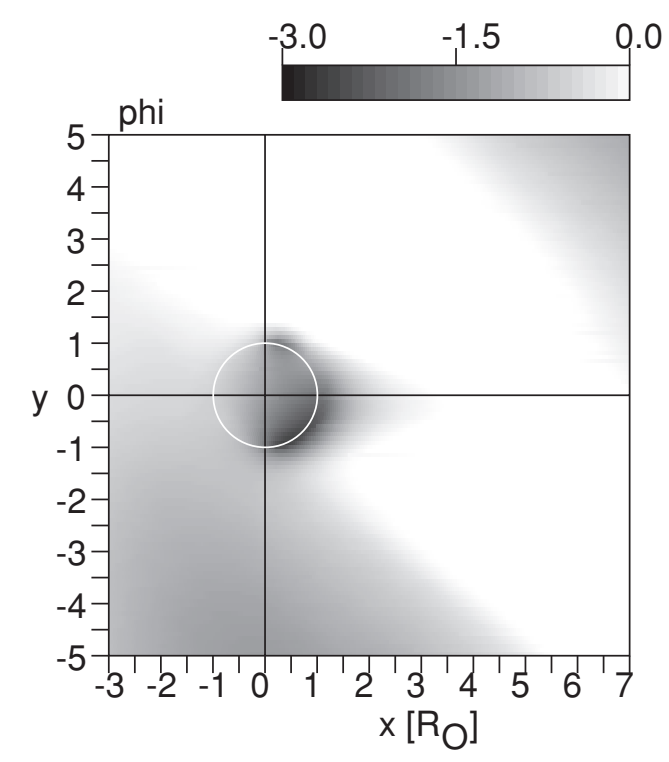

Fig. 4. A gray scale map of the electric potential $\phi$ around the obstacle for simulation run \#1, normalized with $\phi_{0} \equiv m_{\mathrm{e}} v_{\mathrm{e}}^{2} / q_{0}$. The dark color indicates the negative potential. The negative potential on the downstream side surface of the obstacle is asymmetric with respect to the $x$ axis.

A potential drop was found on the downstream side of the obstacle. It extends as far as $3.3 R_{\mathrm{O}}$ due to the electrons in the central wake. On the nightside surface of the obstacle, the largest potential drop is found in the negative- $y$ sector at which the solar wind magnetic field is perpendicular to the surface of the obstacle. The potential drop is as large as $\phi \sim-2.8 \phi_{0}$ at $x \sim 0.7 R_{\mathrm{O}}$ on the negative- $y$ sector, while on the positive- $y$ sector, the maximum potential drop is $\phi \sim-1.7 \phi_{0}$ at around $x \sim 0.3 R_{\mathrm{O}}$, where $\phi_{0} \equiv m_{0} v_{\mathrm{e}}^{2} / q_{0}$. The magnitude of the potential drop is comparable to the floating potential of an artificial satellite in the solar wind plasma without photoelectrons (e.g., Fahleson, 1967).

The weak negative potential in the lower left area of Fig. 4 is due to the enhancement of the electron density as 

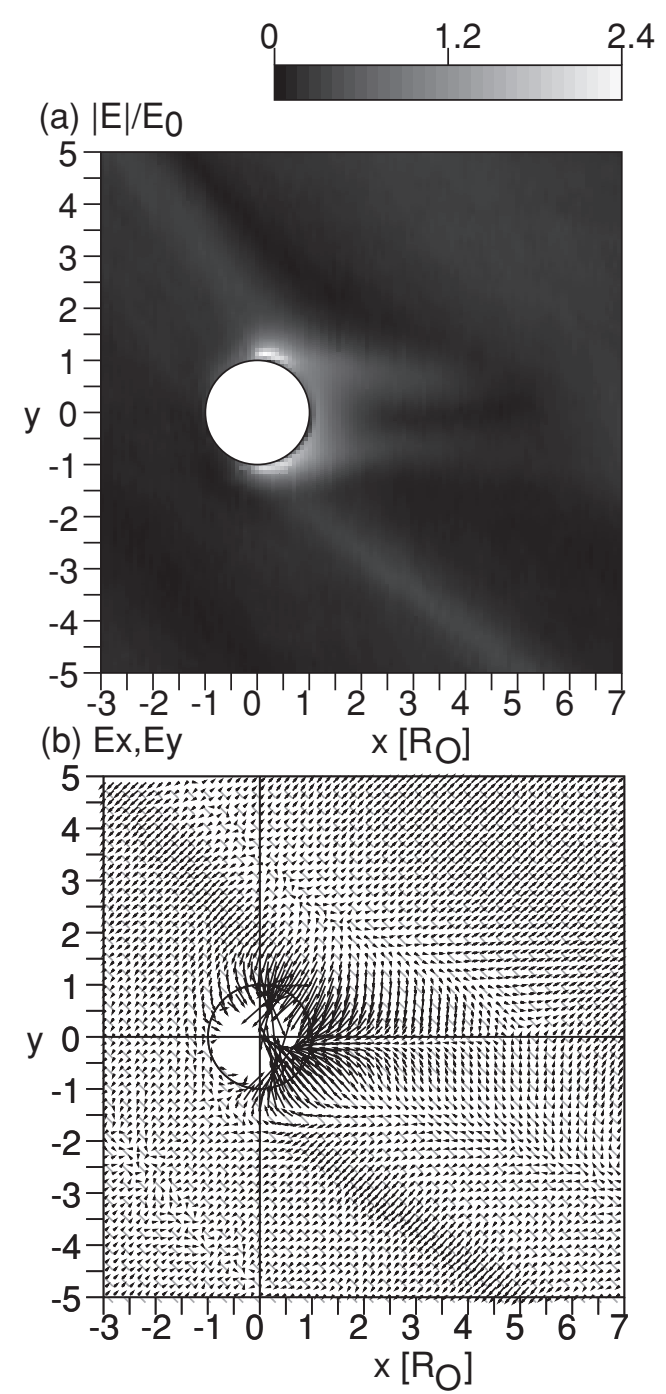

Fig. 5. (a) A gray scale plot of the magnitude of the electric field and (b) a vector presentation of the direction of the electric field overlaid on the magnetic field (gray bars), obtained from simulation run \#1. The solar wind magnetic field deviates $-45^{\circ}$ from the $x$-axis.

observed in Fig. 1. The negative potential on the upper right area is not real; it is due to the periodic boundary condition of the simulation.

Figure 5(a) shows the magnitude of the electric field $|E|=\sqrt{E x^{2}+E y^{2}}$ caused by the distribution of the electric charges around the obstacle. It does not include the solar wind electric field generated by the motion of the solar wind magnetic field past the obstacle $\left(-\mathbf{V}_{\mathrm{sw}} \times \mathbf{B}\right.$ field $)$ in the $z$-direction.

As well as in the non-magnetized solar wind (Kimura and Nakagawa, 2008), we observe the electric fields at the wake boundary. They are asymmetric with respect to the $x$-axis.

The most intense electric field is found at the terminator, where the neutral surface exposed to the solar wind and the negatively-charged surface on the downstream side are adjacent to each other. The magnitude is as large as $2.0 E_{0}$ on the positive- $y$ side, where $E_{0} \equiv m_{\mathrm{e}} v_{\mathrm{e}} \omega_{\mathrm{p}} / q_{0}$. The electric field on the negative- $y$ side occupies a larger area but the magnitude is somewhat weaker $\left(E \sim 1.6 E_{0}\right)$ than the positive- $y$ side, because the potential gradient is not so large

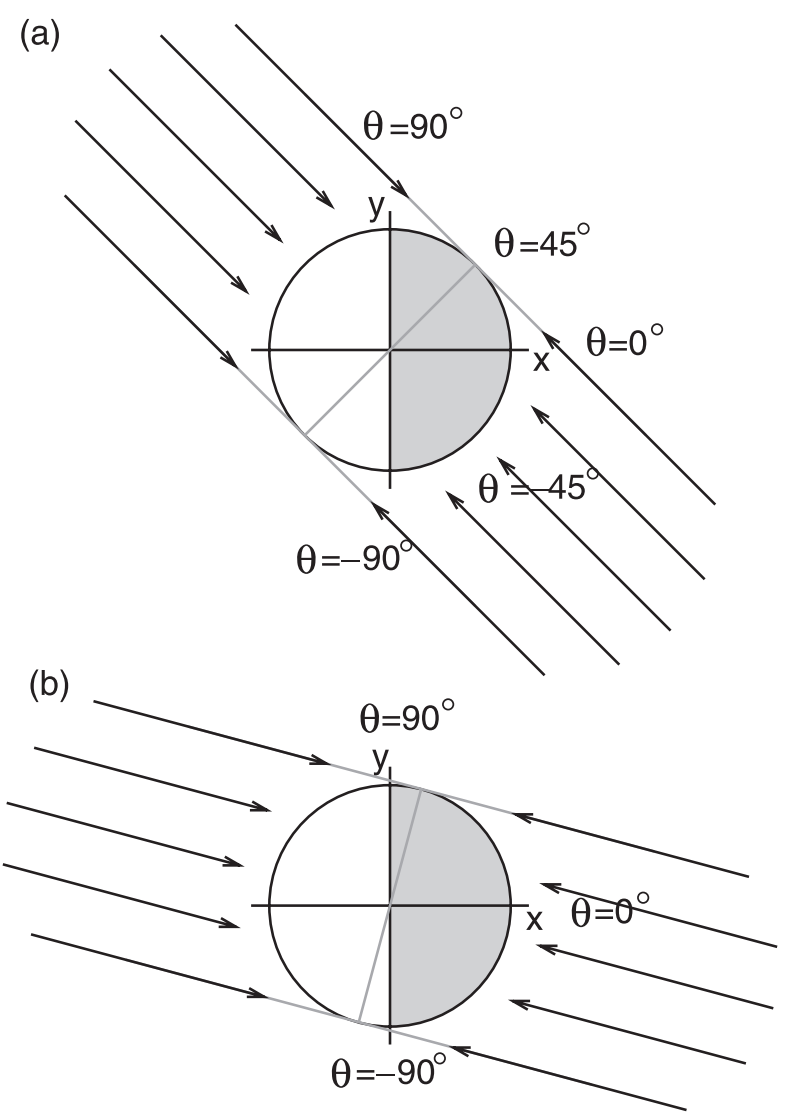

Fig. 6. A schematic illustration of the electrons' access to the surface of the obstacle in the solar wind with the magnetic field (a) $\theta_{\mathrm{B}}=-45^{\circ}$ and (b) $\theta_{\mathrm{B}}=-15^{\circ}$ measured from the $x$ axis. The solar wind flows from left to the right (in the $x$ direction).

due to the larger extent of negative charge on the surface.

Corresponding to the enhancements of the electron density, streaks of enhanced electric fields are also recognized in Fig. 5(a). In the vicinity of the obstacle, the electric field has a component parallel to the magnetic field as shown in Fig. 5(b). This accelerates the electrons in the direction parallel to the magnetic field.

\section{Discussion}

\subsection{Magnetic field control of the surface charging}

In contrast with the non-magnetized solar wind case, in which the solar wind electrons are able to access the nightside surface of the obstacle freely from any direction (Kimura and Nakagawa, 2008), the motion of the electrons in the magnetized solar wind case is controlled by the solar wind magnetic field. The electrons are confined within a Larmour radius to the magnetic field line and we can moreor-less approximate electron flow to be along the magnetic field. Only the electrons on the magnetic field lines that connect with the obstacle can contribute to surface charging.

Figure 6(a) illustrates an example of the magnetic field with the angle $\theta_{\mathrm{B}}=-45^{\circ}$ measured from the flow direction of the solar wind. The thermal electrons come from the upper left, or the lower right, direction along the field lines. The electron flux arriving at the obstacle per unit area on the surface should be largest at around $-45^{\circ}$ and 


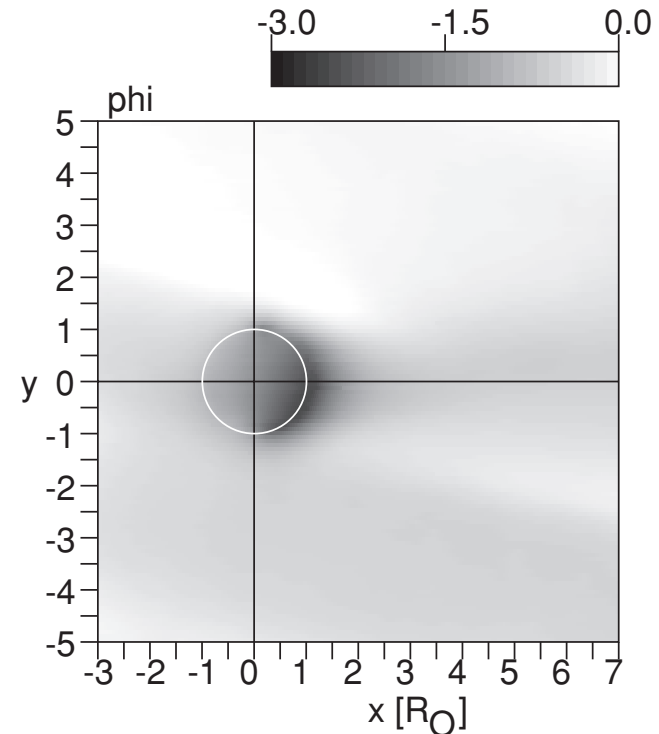

Fig. 7. The electric potential $\phi$ for the simulation run $\# 2$, in which the magnetic field direction is $\theta_{\mathrm{B}}=-15^{\circ}$ measured from the $x$-axis. The potential is normalized with $\phi_{0} \equiv m_{\mathrm{e}} v_{\mathrm{e}}^{2} / q_{0}$.

$135^{\circ}$, where the magnetic field is perpendicular to the surface. (Precisely speaking, the positions shift slightly due to the solar wind bulk velocity.) As we assume the body to be non-conducting, the electric charge accumulates at the position of impact and does not migrate on the surface. The electric charge on the upstream-side surface can be easily neutralized by the incoming solar wind ions and only the electrons that reach the downstream-side surface contribute to the charge accumulation. On the positive- $y$ side, a small area in the vicinity of the terminator $\left(45^{\circ}<\theta<90^{\circ}\right)$ collects electrons from the upstream side. A small number of field lines are connected with the positive- $y$ side and they intersect the surface at an oblique angle. On the other hand, a large area of the negative- $y$ side and a part of the positive- $y$ side extending from $-90^{\circ}$ to $45^{\circ}$ collects a larger number of electrons, with the maximum near $\theta \sim-45^{\circ}$. Thus, the surface charging and the potential drop are expected to be large on the negative- $y$ side. On the positive- $y$ side, the potential drop should be smaller and shifted to the terminator. The negative charging would vanish at around $\theta \sim 45^{\circ}$ where the magnetic field lines do not intersect the surface. These features are consistent with the electric potential shown in Fig. 4.

Figure 6(b) shows an example of the magnetic field with $\theta_{\mathrm{B}}=-15^{\circ}$. In this case, a wide range of the downstreamside surface $-90^{\circ}<\theta<75^{\circ}$ is covered by the electron flux coming from downstream, and only a small area near the terminator of the positive- $y$ side is hit by the electrons from upstream. Figure 7 shows the result of simulation run $\# 2$ for the magnetic field direction $\theta_{\mathrm{B}}=-15^{\circ}$. Compared with Fig. 4, the position of the maximum potential drop has shifted to the center of the void, consistent with Fig. 6(b). The asymmetry is less significant because a wide area of the downstream side is exposed to the electron flux from downstream. The negative potential of the central wake extends far beyond $7 R_{\mathrm{O}}$, as in the non-magnetized solar wind case (figure 2 of Kimura and Nakagawa, 2008).

The asymmetry of the electric field structure vanishes when the magnetic field is parallel, or perpendicular to the solar wind flow. The asymmetry is caused by the oblique magnetic field; it would be most significant at the heliospheric distance of $1 \mathrm{AU}$, where $\theta \sim-45^{\circ}$ on average. On the Moon, for example, the negative charging and the maximum potential drop are expected to be shifted to the duskside of the nightside surface. Since the solar wind magnetic field is variable, there would be sudden changes of charge and discharge as observed by Apollo missions (Colwell et al., 2007, and references therein) at abrupt changes of the magnetic field direction.

\subsection{Streaks of the enhanced electron density}

In the absence of photoelectrons, the electrically-neutral surface on the upstream side of the non-magnetized body is not an obstacle as seen from the electrons of the upstream solar wind. Only the negatively-charged surface is the obstacle that expels the electrons. Figure 8 shows the electric potential as seen from the electrons (that is, reversed in sign) plotted against the distance along a magnetic field line. Initially, the magnetic field line upstream of the nonmagnetized body (which crosses the $x$-axis at $x=-2$ in Fig. 8) is nearly equipotential. A potential difference as large as $2 \phi_{0}$ appears on the field line at the terminator (which crosses the $x$-axis at $x=-1$ in Fig. 8). The electrons that cannot climb up the potential difference are accelerated away from the terminator by the electric field component parallel to the magnetic field, and flow down along the field line. The electrons, once accelerated, keep going along the field line although the potential gap is restricted to a small area near the obstacle.

We can estimate the speed of the electron flow $v_{\|}$along the magnetic field line to be $v_{\|} \sim 2 v_{\mathrm{e}}$. The electrons flow down along the magnetic field line, while the field line is convected down at the solar wind speed $v_{\mathrm{sw}}=0.25 v_{\mathrm{e}}$. Combining the thermal velocity $\left(v_{||} \cos \theta_{\mathrm{B}}, v_{||} \sin \theta_{\mathrm{B}}, 0\right)$ with the convection velocity $\left(v_{\mathrm{sw}}, 0,0\right)$ as illustrated in Fig. 9, we obtain the flow direction $-40^{\circ}$ measured from the direction of the solar wind flow. On the positive- $y$ side, the electrons flow along the magnetic field line against the solar wind bulk flow with the velocity $\left(-v_{||} \cos \theta_{\mathrm{B}},-v_{||} \sin \theta_{\mathrm{B}}, 0\right)$ and the flow direction as seen from the obstacle is $129^{\circ}$ from the solar wind direction. These are consistent with the direction of the streaks of the electron enhancements, $-37^{\circ}$ and $131^{\circ}$, as has been observed in Fig. 1.

In the case of the magnetic field whose direction is $\theta_{\mathrm{B}}=$ $-15^{\circ}$ from the $x$-axis, the flow directions of the accelerated electrons are calculated to be $-13^{\circ}$ and $163^{\circ}$. Figure 10 shows the electron density obtained from the simulation run $\# 2$ with $\theta_{\mathrm{B}}=-15^{\circ}$. The electron enhancements streak in the direction $-14^{\circ}$ measured from the $x$-axis on the negative- $y$ side and in the direction $160^{\circ}$ on the positive- $y$ side, although this is rather faint due to the very small area of negative charge on the surface near the terminator on the positive- $y$ side. They agree with the above expectations.

\subsection{Dependence on the Debye length}

In general, the spatial extent of the electric field caused by the surface charging is of the order of the Debye length. In this paper, a Debye length $\lambda_{\mathrm{D}}$ as large as $0.25 R_{\mathrm{O}}$ has been 


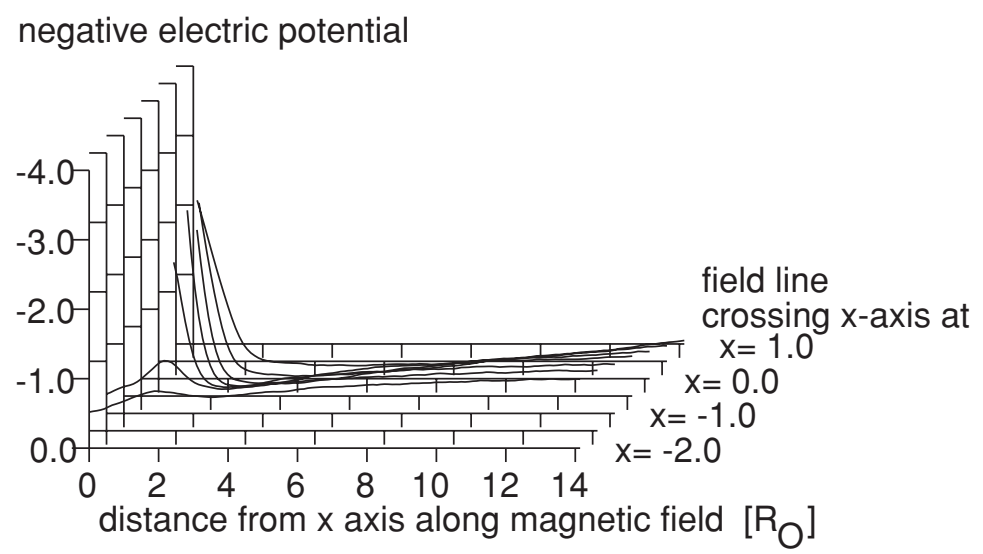

Fig. 8. Reversed electric potential plotted versus the distance along each magnetic field line on the negative- $y$ side. Note that the vertical line is reversed to indicate the barrier for the electrons. The potential is normalized with $\phi_{0} \equiv m_{\mathrm{e}} v_{\mathrm{e}}^{2} / q_{0}$. This result is from simulation run \#1.

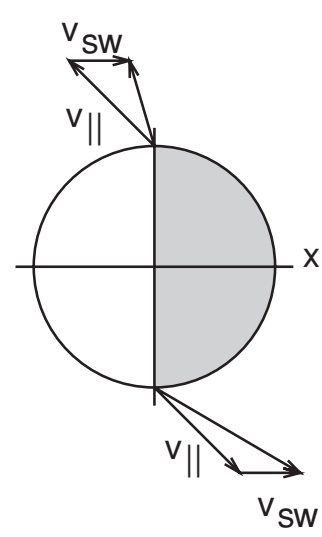

Fig. 9. A schematic illustration of the motion of electrons accelerated by the surface potential.

employed. There might be a concern that the effect of the surface charging is limited for an object whose radius $R_{\mathrm{O}}$ is much larger with respect to the Debye length.

Figure 11 shows the result of the simulation run \#3, for which the Debye length is reduced to be $0.125 R_{\mathrm{O}}$ by slowing down the electron thermal speed. The solar wind bulk speed and the ion thermal speed are also reduced in the same proportion. As expected, the spatial extent of the electron void around the terminator is smaller in Fig. 11(a) than in Fig. 1. On the other hand, the void in the central wake is essentially the same.

The asymmetry of the potential structure is clearer in Fig. 11(b) than in Fig. 4. The largest potential drop is $\phi \sim-3 \phi_{0}$. (Note that $\phi_{0}$ is also reduced by slowing down the electron thermal speed $v_{\mathrm{e}}$.) As the ratio of the potential drop to the electron thermal energy is nearly the same as before, the electrons gain as much flow speed as before and the streaks of electron enhancement appear in Fig. 11(a).

The potential drop in the downstream wake extends far beyond $7 R_{\mathrm{O}}$ in Fig. 11(b), differently from the larger Debye length case in Fig. 4. The relative importance of the wake potential to the surface charging increases for a larger scale obstacle.

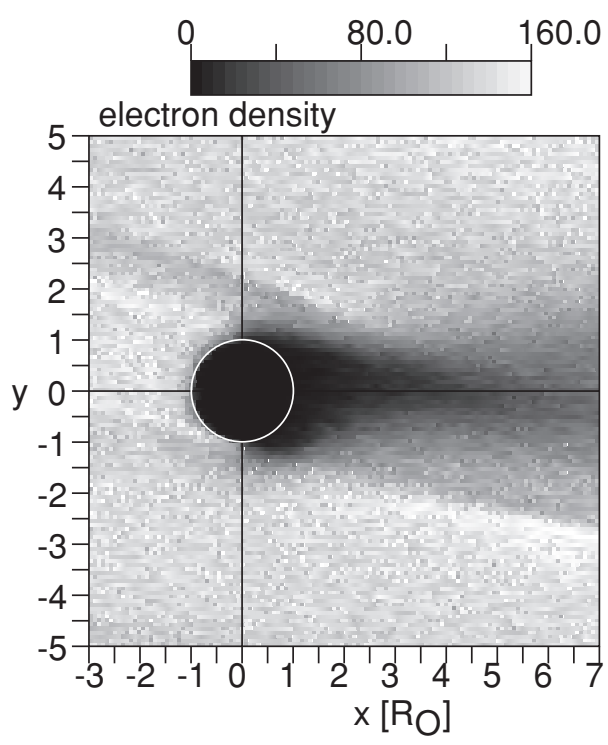

Fig. 10. The electron density $n_{\mathrm{e}}$ obtained from the simulation run \#2. The solar wind flows from left to right. The solar wind magnetic field is $-15^{\circ}$ from the $x$-axis.

\subsection{Weaker magnetic field case}

We have employed an intense magnetic field $\Omega_{\mathrm{e}}=12 \omega_{\mathrm{p}}$ and there might be a concern that the control by the magnetic field is too strong in these simulations. So we carried out another simulation run (\#4) in which the magnitude of the magnetic field is as small as $\Omega_{\mathrm{e}}=0.75 \omega_{\mathrm{p}}$. In this case, the ion Larmour radius $r_{\mathrm{iL}}$ is as large as $7.54 R_{\mathrm{O}}$, i.e. the ions are almost non-magnetized, and the electron Larmour radius $r_{\mathrm{eL}}$ is $0.23 R_{\mathrm{O}}$, larger than the Debye length $\lambda_{\mathrm{D}}=0.125 R_{\mathrm{O}}$. Figure 12 shows the electric potential obtained from run \#4. The asymmetry of the potential structure is recognized, although it is not as clear as in Fig. 11(b), due to the large electron Larmour radius. It shows that the magnetic field control of the surface charging of the nonmagnetized obstacle is significant, as long as the electron Larmour radius is smaller than the size of the obstacle.

\subsection{Comparison with observations at the Moon}

Limitation of the scale size of the obstacle with respect to the Debye length, together with the absence of photoemis- 

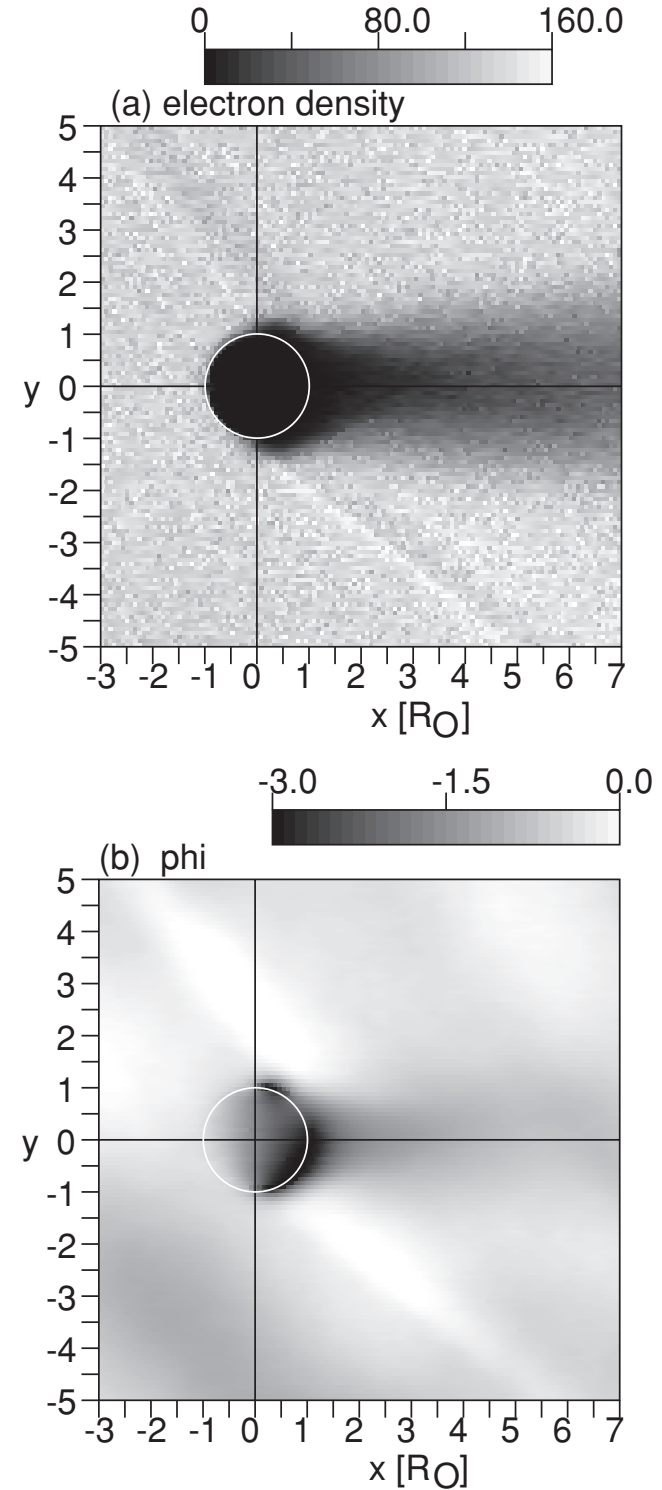

Fig. 11. (a) The electron density $n_{\mathrm{e}}$ and (b) the electric potential $\phi$ for the reduced Debye length case $\lambda_{\mathrm{D}}=0.125 R_{\mathrm{O}}$, obtained from the simulation run \#3 at time $t=9.0 \times 10^{2} \omega^{-1}$ (180000 steps) when the solar wind flow has swept the simulation domain twice. The potential is normalized with $\phi_{0} \equiv m_{\mathrm{e}} v_{\mathrm{e}}^{2} / q_{0}$.

sion, prohibits us from making a direct comparison of the simulation result with the observations made at the Moon. Too small a ratio of the obstacle size to the Debye length magnifies the effect of surface charging with respect to the potential drop at the wake boundary. Nevertheless, some aspects of the model can be compared with the lunar data. Such a comparison would help elucidate what aspects of the model are appropriate for all scale sizes of objects and which are more limited to smaller objects.

As we have seen in Section 4.2, the potential drop at the terminator is of the order of $2 \phi_{0}$, which corresponds to 60 $80 \mathrm{~V}$ for the typical solar wind electrons having a thermal energy of 15-20 eV. This is consistent with the Apollo SIDE observation of $70 \mathrm{eV}$ ions accelerated by the negative lunar surface potential (Freeman and Ibrahim, 1975) and a surface potential as low as $-100 \mathrm{~V}$ on some terminator

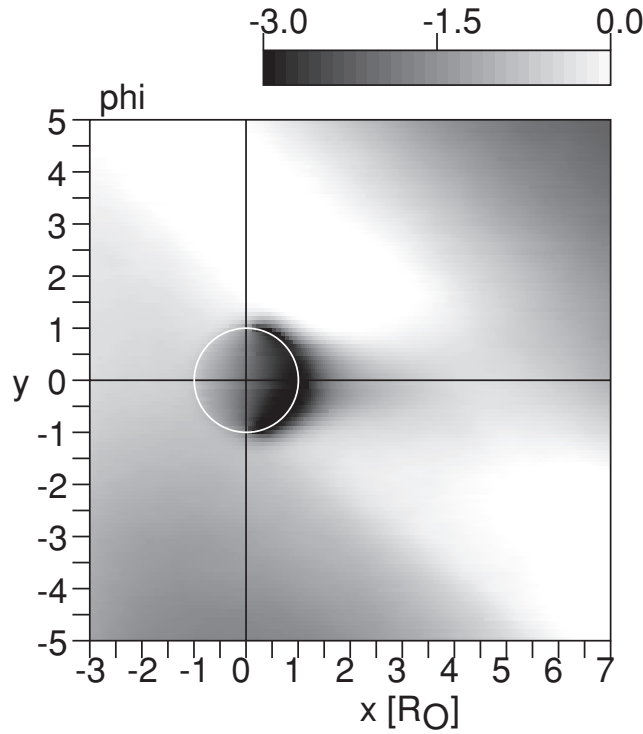

Fig. 12. Electric potential in a weak solar wind magnetic field, obtained from the simulation run \#4, at time $t=9.4 \times 10^{2} \omega^{-1}$ (60000 steps) when the solar wind flow has swept the simulation domain a little more than twice.

crossings (Lindeman et al., 1973).

The largest potential drop on the nightside surface of the obstacle, $3 \phi_{0}$, which corresponds to $90-120 \mathrm{~V}$, is consistent with a lunar surface potential of $-120 \mathrm{~V}$ inferred from the Lunar Prospector observation of the electrons at an altitude of 20-40 km (Halekas et al., 2002), but somewhat smaller than the newly found potential drop of $-200 \mathrm{~V}$ near the edge of the wake (Halekas et al., 2008). Halekas et al. (2008) also reported that the surface potential drop with respect to the local plasma is smaller in the central wake than near the wake boundary. No such signature is found in this simulation. Halekas et al. (2008) attributed this to secondary electrons, which are not included in the present simulation.

The minimum electron density obtained by Lunar Prospector in the lunar wake (figure 6 of Halekas et al., 2005) appears to be shifted slightly to the duskside, consistent with the result of the present simulations. This is likely, because the magnetic field lines of the average IMF at $1 \mathrm{AU}$ are perpendicular to the dusk-to-night surface of the moon.

It is difficult to apply the wake potential obtained from the simulation with a large Debye length to the lunar observations. In the classical theory of a plasma expansion into a vacuum (Samir et al., 1983), the electrons were thought to rush into the void faster than the ions due to the faster thermal speed. However, in the present simulation with nightside surface charging, the electrons are retarded by the negative charging of the downstream-side surface, and cannot precede the ions. Figure 13(a) shows the ion and electron densities for several distances from the obstacle, obtained from simulation run \#3. In the vicinity of the obstacle $\left(x=1 R_{\mathrm{O}}\right)$, the ions enter the void faster than the electrons, producing a positive excess of charge in the vicinity of the wake boundary which affects the potential structure as observed in the top panel of Fig. 13(b). Such an effect of 
(a) ion density

160 electron density $x=1.0$ Ro

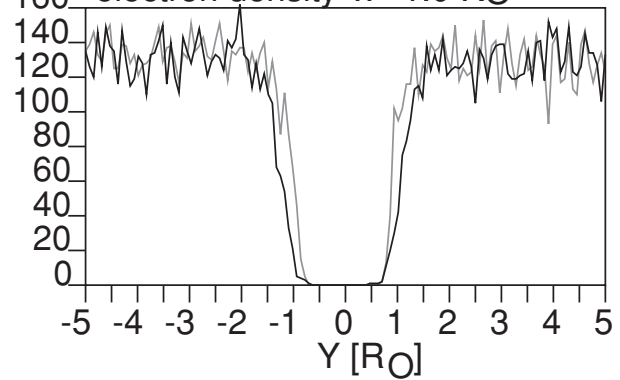

ion density

160 electron density $\mathrm{x}=2.0 \mathrm{Ro}$

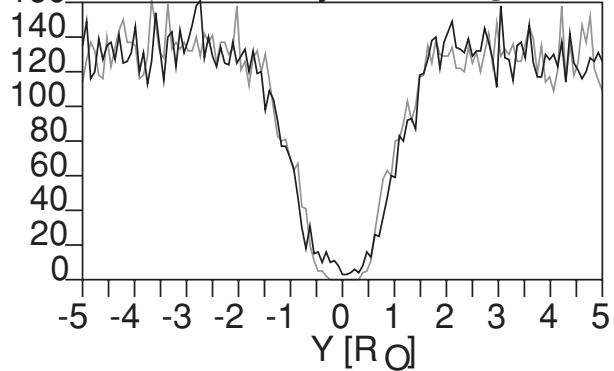

ion density

160 electron density $x=3.0$ Ro

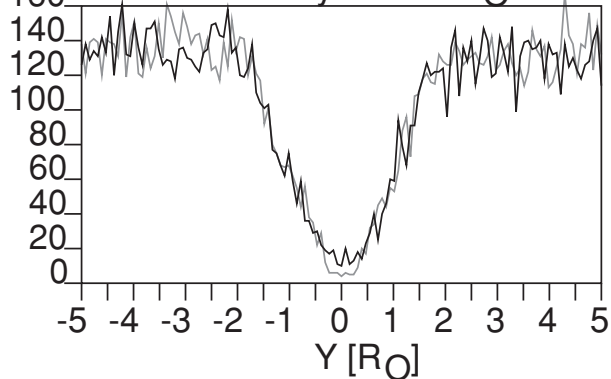

ion density

160 electron plensity $x=4.0$ Ro

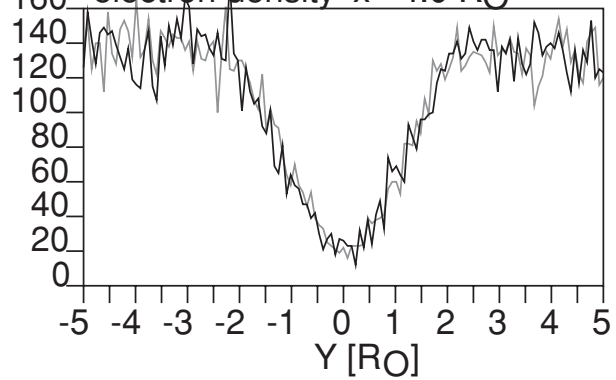

(b)
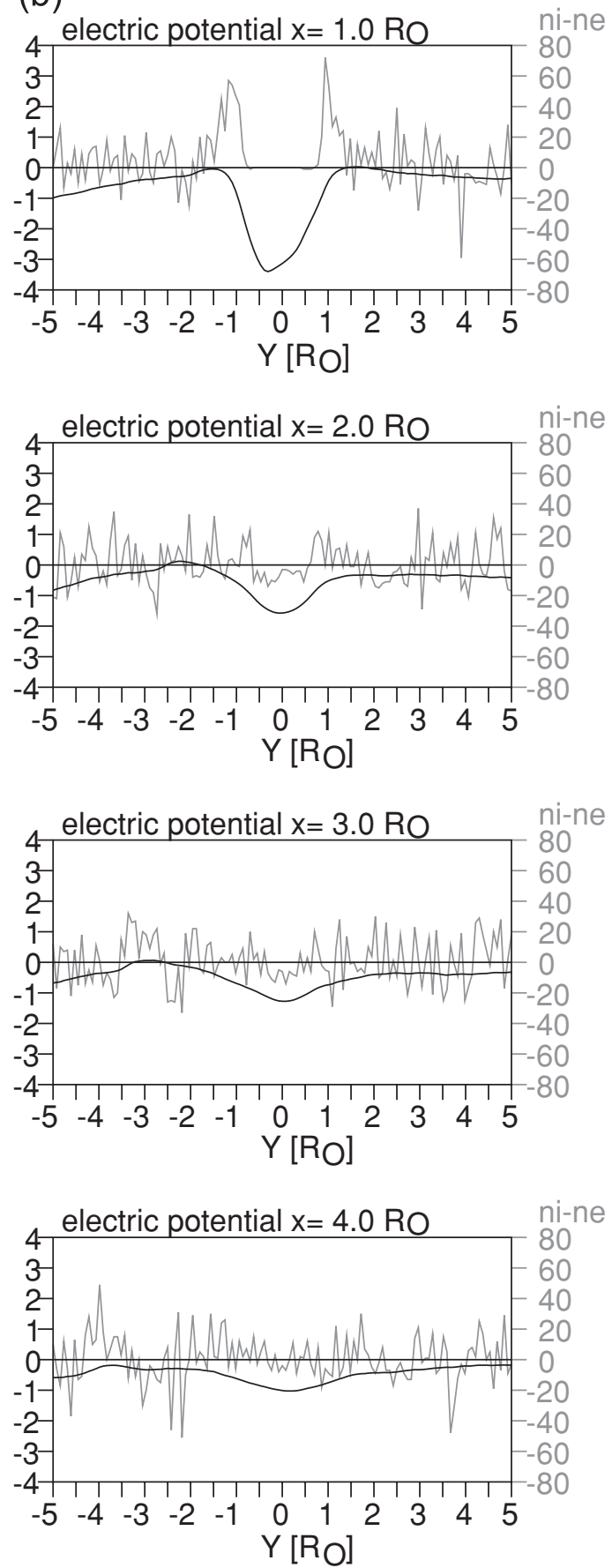

Fig. 13. (a) The ion and electron densities $n_{\mathrm{i}}, n_{\mathrm{e}}$ (gray and black curves, respectively) and (b) the electric potential $\phi$ (black) overlaid on the density difference $n_{\mathrm{i}}-n_{\mathrm{e}}$ (gray), obtained from the simulation run \#3 at time $t=9.4 \times 10^{2} \omega^{-1}$, the same as in Fig. 11 . The potential is normalized with $\phi_{0} \equiv m_{\mathrm{e}} v_{\mathrm{e}}^{2} / q_{0}$.

the surface charging should be more limited within a small area.

A negative excess of charge is found in the central wake at $x=2-3 R_{\mathrm{O}}$ in Fig. 13 and disappears at $4 R_{\mathrm{O}}$. At $x=3 R_{\mathrm{O}}$, well beyond the Debye length from the obstacle (although the Debye length becomes large in a low density plasma), the potential drop in the central wake with respect to the ambient solar wind is about $0.5 \phi_{0}-1 \phi_{0}$. If we assume that this is the wake potential and that the wake potential is essentially independent of the Debye length, as long as the ratio of the thermal speeds to the solar wind speed is kept constant, it is not necessary to evaluate it in terms of the electron thermal energy $m_{\mathrm{e}} v_{\mathrm{e}}^{2} / q_{0}$, but rather, we can convert it directly into volts using $v_{\mathrm{e}}=0.05 \mathrm{c}$ for the simulation run \#3. It is calculated to be $0.64-1.3 \mathrm{kV}$. This is much stronger than it appears in Fig. 11 in which the surface charging effect is magnified. Although this is a very rough estimation, it is of the same order as the potential of $-442 \mathrm{~V}$ estimated from the WIND observation of backstreaming electrons (Farrell et al., 1996) and $-480 \mathrm{~V}$ estimated from NOZOMI observation of counterstreaming electrons (Futaana et al., 2001). 
This simulation reproduced the streaks of the electron enhancement along the magnetic field line on the same hemisphere as the electron enhancement detected by Kaguya LRS/WFC-H, but with a slight difference in the location. This might be due to the effect of surface charging, or the absence of photoelectrons in this simulation. Photoelectrons might affect the position of the first contact of the magnetic field line with the negative surface density. At present, we cannot conclude that they are the same phenomena or not.

The horizontal ion entry along the magnetic field lines as reported by Futaana et al. (2010) is not observed in the present simulation.

\section{Conclusion}

A two-dimensional, electromagnetic particle-in-cell simulation has revealed that the solar wind magnetic field controls the direction of the thermal flow of the solar wind electrons onto a non-magnetized, non-conducting obstacle immersed in the solar wind flow. The accumulation of the negative charge on the downstream surface of the obstacle is largest at the position where the solar wind magnetic field is perpendicular to the surface. The asymmetry of the surface charging causes an asymmetry of electric potential structure.

In the absence of photoemission, the solar wind electrons on the equipotential magnetic field line suddenly gain potential energy on arrival of the field line at the negatively charged surface at the terminator. The electrons begin to flow down the field line away from the obstacle, forming streaks of enhanced electron density. It is likely that the photoelectrons and secondary electrons, that were not included in the present simulation, modify the surface charging near the terminator and the position of the electron enhancements around the terminator. Their inclusion will be necessary in future studies.

Acknowledgments. The author thanks M. Iizima for his suggestion concerning the initial condition of the electric field. The authors are grateful to the reviewers for their valuable comments that improved the manuscript. This study was supported by the JSPS grant-in-aid for scientific research project 21540461.

\section{References}

Birch, P. C. and S. C. Chapman, Correction to "Particle-in-cell simulations of the lunar wake with high phase resolution", Geophys. Res. Lett., 28, 2669, 2001.

Birch, P. C. and S. C. Chapman, Two dimensional particle-in-cell simulations of the lunar wake, Phys. Plasmas, 9, 1785-1789, 2002.

Birdsall, C. K. and A. B. Langdon, Plasma Physics via Computer Simulation, McGraw-Hill, New York, 1985.

Colwell, J. E., S. Batiste, M. Horányi, S. Robertson, and S. Sture, Lunar surface: dust dynamics and regolith mechanics, Rev. Geophys., 45, RG2006, doi:10.1029/2005RG000184, 2007.

Fahleson, U., Theory of electric field measurements conducted in the magnetosphere with electric probes, Space Sci. Rev., 7, 238-262, 1967.

Farrell, W. M., R. J. Fitzenreiter, C. J. Owen, J. B. Byrnes, R. P. Lepping, K. W. Ogilvie, and F. Neubauer, Upstream ULF waves and energetic electrons associated with the lunar wake: Detection of precursor activity, Geophys. Res. Lett., 23, 1271-1274, 1996.
Farrell, W. M., M. L. Kaiser, J. T. Steinberg, and S. D. Bale, A simple simulation of a plasma void: Applications to Wind observations of the lunar wake, J. Geophys. Res., 103, 23635-23653, 1998.

Freeman, J. W. and M. I. Ibrahim, Lunar electric fields, surface potential and associated plasma sheaths, Moon, 14, 103-114, 1975.

Futaana, Y., S. Machida, T. Saito, A. Matsuoka, and H. Hayakawa, Counterstreaming electrons in the near vicinity of the moon observed by plasma instruments on board NOZOMI, J. Geophys. Res., 106, 1872918740, 2001.

Futaana, Y., S. Barabash, M. Wieser, M. Holmstrom, A. Bhardwaj, M. B. Dhanya, R. Sridharan, P. Wurz, A. Schaufelberger, and K. Asamura, Protons in the near lunar wake observed by the SARA instrument on board Chandrayaan-1, J. Geophys. Res., 115, A10248, doi:10.1029/2010JA015264, 2010.

Guio, P. and H. L. Pécseli, Phase space structures generated by an absorbing obstacle in a streaming plasma, Geophys. Res. Lett., 31, L03806, 2004.

Guio, P. and H. L. Pécseli, Phase space structures generated by absorbing obstacles in streaming plasmas, Ann. Geophys., 23, 853-865, 2005.

Halekas, J. S., D. L. Mitchell, R. P. Lin, L. L. Hood, M. N. Acunã, and A. B. Binder, Evidence for negative charging of the lunar surface in shadow, Geophys. Res. Lett., 29(77), doi:10.1029/2001GL014428, 2002.

Halekas, J. S., R. P. Lin, and D. L. Mitchell, Inferring the scale height of the lunar nightside double layer, Geophys. Res. Lett., 30, PLA1, 2117 , doi:10.1029/2003GL018421, 2003.

Halekas, J. S., S. D. Bale, D. L. Mitchell, and R. P. Lin, Electrons and magnetic fields in the lunar plasma wake, J. Geophys. Res., 110, A07222, doi:10.1029/2004JA010991, 2005.

Halekas, J. S., G. T. Delory, R. P. Lin, T. J. Stubbs, and W. M. Farrell, Lunar Prospector observations of the electrostatic potential of the lunar surface and its response to incident currents, J. Geophys. Res., 113, A09102, doi:10.1029/2008JA013194, 2008.

Kimura, S. and T. Nakagawa, Electromagnetic full particle simulation of the electric field structure around the moon and the lunar wake, Earth Planets Space, 60, 591-599, 2008.

Lindeman, R., J. W. Freeman, Jr., and R. R. Vondrak, Ions from the lunar atmosphere, Geochim. Cosmochim. Acta, Suppl. (Proceedings of the Lunar Science Conference, vol. 4), 3, 2889-2896, 1973.

Nishino, M. N., K. Maezawa, M. Fujimoto, Y. Saito, S. Yokota, K. Asamura, T. Tanaka, H. Tsunakawa, M. Matsushima, F. Takahashi, T. Terasawa, H. Shibuya, and H. Shimizu, Pairwise energy gain-loss feature of solar wind protons in the near-Moon wake, Geophys. Res. Lett., 36, L12108, doi:10.1029/2009GL039049, 2009a.

Nishino, M. N., M. Fujimoto, K. Maezawa, Y. Saito, S. Yokota, K. Asamura, T. Tanaka, H. Tsunakawa, M. Matsushima, F. Takahashi, T. Terasawa, H. Shibuya, and H. Shimizu, Solar-wind proton access deep into the near-Moon wake, Geophys. Res. Lett., 36, L16103, doi:10.1029/2009GL039444, 2009b.

Saito, Y., S. Yokota, K. Asamura, T. Tanaka, R. Akiba, M. Fujimoto, H. Hasegawa, H. Hayakawa, M. Hirahara, M. Hoshino, S. Machida, T. Mukai, T. Nagai, T. Nagatsuma, M. Nakamura, K.-I. Oyama, E. Sagawa, S. Sasaki, K. Seki, and T. Terasawa, Low-energy charged particle measurement by MAP-PACE onboard SELENE, Earth Planets Space, 60, 375-385, 2008.

Samir, U., K. H. Wright, Jr., and N. H. Stone, The expansion of a plasma into a vacuum: Basic Phenomena and processes and applications to space plasma physics, Rev. Geophys. Space Sci., 21, 1631-1646, 1983.

Schubert, G. and B. R. Lichtenstein, Observations of moon-plasma interactions by orbital and surface experiments, Rev. Geophys. Space Phys., 12, 592-626, 1974.

Tsunakawa, H., H. Shibuya, F. Takahashi, H. Shimizu, M. Matsushima, A. Matsuoka, S. Nakazawa, H. Otake, and Y. Iijima, Lunar magnetic field observation and initial global mapping lunar magnetic anomalies by MAP-LMAG onboard SELENE (Kaguya), Space Sci. Rev., 154, 219$251,2010$.

T. Nakagawa (e-mail: nakagawa@tohtech.ac.jp) and S. Kimura 